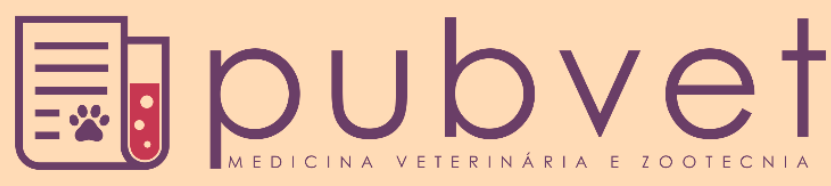

https://doi.org/10.31533/pubvet.v15n12a987.1-7

\title{
Equívocos ao se antropomorfizar a alimentação dos animais de companhia
}

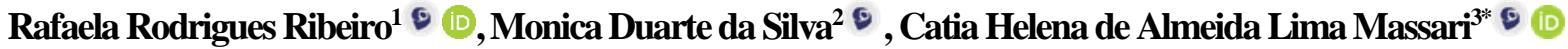 \\ ${ }^{1}$ Graduanda em Medicina Veterinária da Universidade São Judas Tadeu, São Paulo-SP, Brasil \\ ${ }^{2}$ Doutoranda no Programa de Pós-Graduação em Anatomia dos Animais Domésticos e Silvestres, Universidade de São Paulo, São Paulo-SP, Brasil \\ ${ }^{3}$ Professora colaboradora do Departamento de Cirurgia, Faculdade de Medicina Veterinária e Zootecnia, Universidade de São Paulo, São Paulo-SP, Brasil \\ *Autora para correspondência, E-mail: catia.massari@usp.br
}

Resumo. O presente trabalho objetiva contribuir para a questão da responsabilidade legal e ética quanto à alimentação adequada para animais de companhia, por meio da análise das obras dos filósofos Singer, Francione e Gilligan. Realizou-se uma revisão de literatura a partir das principais obras filosóficas de autores que discutem a ética e os direitos dos animais, além de artigos científicos atuais e pertinentes ao tema. Verificou-se que, apesar das críticas filosóficas, cães e gatos ainda possuem o status de "coisa" sob a visão jurídica brasileira, ao invés de serem considerados sujeitos de direitos. Dentre os diversos atos abusivos contra os animais, inclui-se a negligência acerca do fornecimento de uma nutrição adequada. Conclui-se que os filósofos estudados propõem novos paradigmas para a relação cada vez mais antropomorfizada entre tutores e seus animais de companhia. E, isso, certamente, reflete em importantes mudanças nos hábitos alimentares dos pets.

Palavras chave: Ética do cuidado, cães, direito dos animais, gatos, nutrição animal

\section{Misconceptions in anthropomorphizing the feeding of pet animals}

\begin{abstract}
The present work aims to contribute to the issue of legal and ethical responsibility regarding adequate feeding for pets, through the analysis of the works of the philosophers Singer, Francione and Gilligan. A literature review was carried out based on the main philosophical works by authors who discuss ethics and animal rights, in addition to current and relevant scientific articles. It was found that, despite philosophical criticism, dogs and cats still have the status of "thing" under the Brazilian legal view, instead of being considered subjects of rights. Among the various abusive acts against animals, there is negligence about providing adequate nutrition. It is concluded that the studied philosophers propose new paradigms for the increasingly anthropomorphic relationship between tutors and their pets. And this certainly reflects important changes in the eating habits of pets.
\end{abstract}

Keywords: Care ethics, dogs, animal rights, cats, animal nutrition

\section{Conceptos erróneos sobre la antropomorfización de la alimentación de las mascotas}

Resumen. El presente trabajo tiene como objetivo contribuir al tema de la responsabilidad legal y ética en la alimentación adecuada de las mascotas, a través del análisis de las obras de los filósofos Singer, Francione y Gilligan. Se realizó una revisión bibliográfica a partir de los principales trabajos filosóficos de autores que discuten la ética y los derechos de los animales, además de artículos científicos actuales y relevantes. Se encontró que, a pesar de la crítica filosófica, los perros y gatos todavía tienen el estatus de "cosa" bajo la visión legal brasileña, en lugar de ser considerados sujetos de derechos. Entre los diversos actos de maltrato contra los animales, se encuentra la negligencia en la provisión de una nutrición 
adecuada. Se concluye que los filósofos estudiados proponen nuevos paradigmas para la relación cada vez más antropomorfizada entre los tutores y sus mascotas. Y, esto, sin duda, se refleja en importantes cambios en los hábitos alimentarios de las mascotas.

Palabras clave: Ética del cuidado, perros, derechos de los animales, gatos, nutrición animal

\section{Introdução}

Segundo o levantamento do Instituto Brasileiro de Geografia e Estatística de 2016, os 52 milhões de cães e os 22 milhões de gatos já ultrapassaram o número de crianças brasileiras menores de 12 anos, compondo as famílias multiespécies (Bontempo, 2005). De domesticações muito distintas, as espécies Canis lupus familiaris e Felis catus apresentam relações extra específicas com o Homo sapiens, datando de, respectivamente, dez mil e seis mil anos atrás. Atuando como agentes de proteção e saneamento ao se alimentarem de restos orgânicos, os cães diferenciaram-se de seus antepassados comuns à espécie Canis lupus, que eram carnívoros estritos, para se tornarem animais onívoros, fenômeno que não ocorreu com o gato doméstico que mantém hábitos alimentares semelhantes ao dos felinos silvestres (Swanson et al., 2003; Zaghini \& Biagi, 2005; Zoran, 2002). Dessa maneira, surgem diversos questionamentos sobre o bem-estar desses animais de companhia que já não mais se inserem em seus habitats e, hoje, comportam-se de maneira adaptada para com o convívio humano.

Além disso, a partir das últimas décadas do século XX, tem-se uma crescente preocupação com o meio ambiente e os impactos antrópicos sobre os ecossistemas, além do sofrimento e do uso dos animais na produção zootécnica. Posto isso, entende-se o bem-estar animal a partir do conceito dos Cinco Domínios, dentre os quais estão incluídos o Domínio da Nutrição, onde se deve ofertar água e alimento suficiente para os animais de acordo com suas necessidades biológicas, e o Domínio do Estado Mental que considera as experiências positivas quanto à nutrição palatável (Buff et al., 2014; Ramos et al., 2020).

Ao selecionar uma dieta para os animais de companhia, os tutores, muitas vezes guiados pelo antropomorfismo, tomam decisões sobre a nutrição de seus pets considerando seus posicionamentos éticos e preocupações com seus estilos de vida, deixando de lado as reais necessidades biológicas das espécies que estão criando (Carciofi \& Jeremias, 2010; Rothgerber, 2014). Tais tutores levam em consideração, também, o custo financeiro a ser dispendido nos pet shops. Todavia, com o crescente número de cães obesos e diabéticos e de felinos malnutridos e anoréxicos, ocorre um considerável impacto da nutrição na saúde desses animais, justo no momento em que o mercado pet mais cresce e uma maior quantidade de alimentos, dietas e receitas, são encontrados nas prateleiras pelos tutores ( $\underline{\mathrm{Li}}$, 2002; Thatcher et al., 2010; Tobie et al., 2015).

Tem-se, então, a importância da responsabilidade legal e moral que os tutores possuem para com seus cães e gatos, principalmente, dentro de uma ética do cuidado onde as relações afetivas são valorizadas nas famílias compostas por pessoas e animais de companhia, compreendidas pelo movimento filosófico conhecido como pós-humanismo. Dentro dessa óptica, devem ser analisadas as principais vantagens e desvantagens de cada dieta e seus impactos na higidez dos animais de companhia e no meio em que vivem, sempre tentando se evitar o especifismo geral e seletivo (Albright, 2002; Francione, 1995; Kuhnen, 2014).

Assim, o presente trabalho objetiva contribuir para a questão da responsabilidade legal e ética quanto à alimentação adequada para animais de companhia, por meio da análise das obras dos filósofos Singer, Francione e Gilligan.

\section{Material e métodos}

Realizou-se uma revisão de literatura a partir das principais obras filosóficas de autores que discutem a ética e os direitos dos animais, sendo eles: Peter Singer; Gary Lawrence Francione e Carol Gilligan (Figura 1). Esta última autora é responsável por propor, pela primeira vez, uma ética das relações familiares a partir da noção de cuidado.

Além dos três livros dos filósofos citados, também foram integrados à revisão de literatura artigos científicos atuais e pertinentes à temática em estudo. Por meio da base de dados do Google Acadêmico, realizou-se uma busca utilizando as seguintes palavras-chave: vegetarian's dilemma, interspecies 
sensemaking, multispecies households, multispecies care, vegan foods for dogs and cats, post-human families, dog-human relations, ética do cuidado, direitos dos animais e família multiespécie. Adotou-se como critério de inclusão os manuscritos publicados nos últimos nove anos (2012-2021). Após análise entre pares (orientada-orientadora), optou-se pela exclusão dos artigos repetidos e, finalmente, chegouse a um resultado de nove trabalhos selecionados. Assim, os seguintes autores incorporaram a presente pesquisa (Charles, 2016; Dias \& Belchior, 2019; Dodd et al., 2019; Gillespie \& Lawson, 2017; Irvine \& Cilia, 2017; Kuhnen, 2014; Mancini et al., 2012; Rothgerber, 2014; Zafalon et al., 2020). Ainda, mais 31 textos científicos foram aqui englobados para se investigar a respeito das necessidades nutricionais das espécies Canis lupus familiaris e Felis catus.

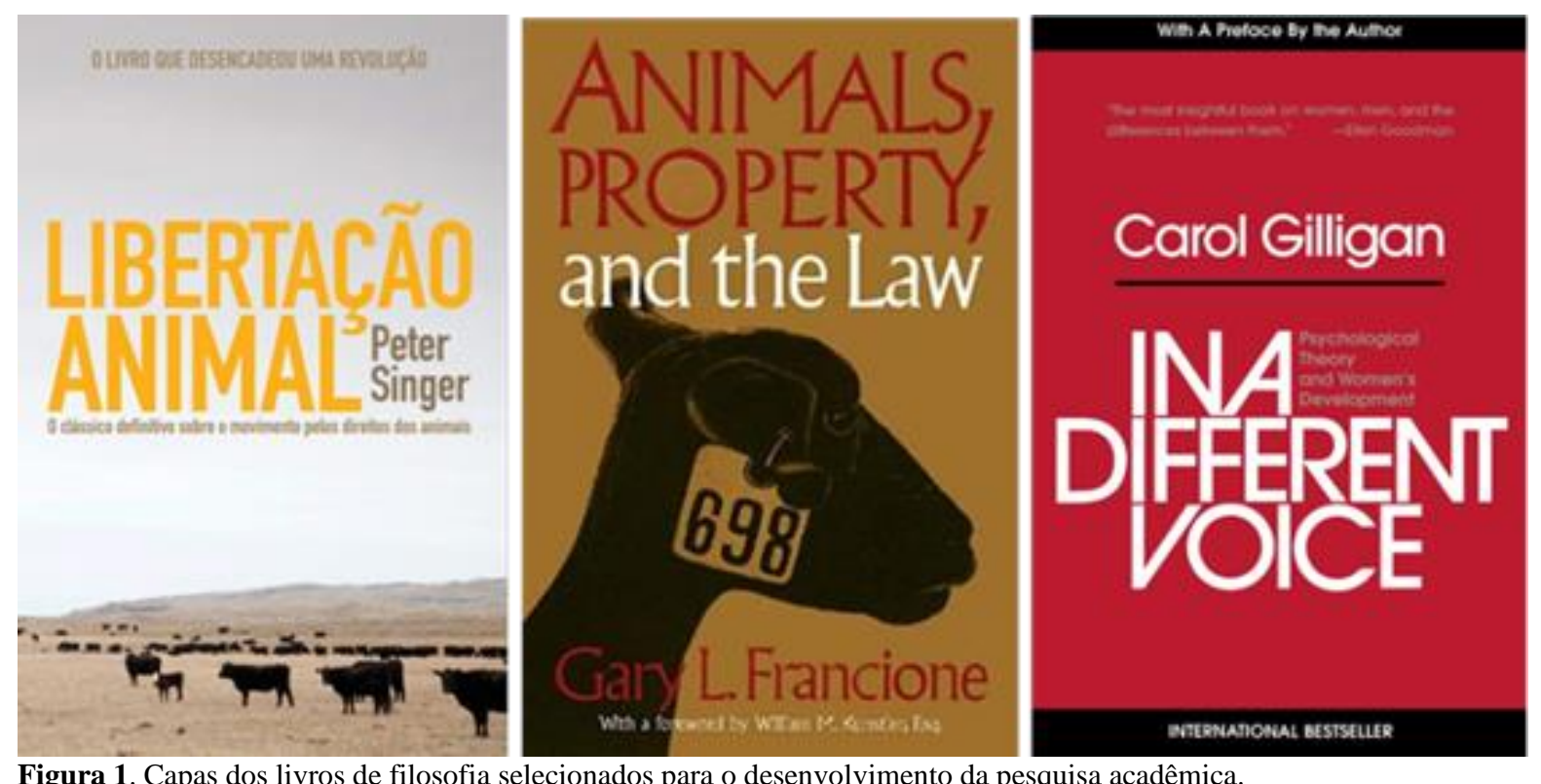

Figura 1. Capas dos livros de filosofia selecionados para o desenvolvimento da pesquisa acadêmica.

\section{Resultados e discussão}

Verificou-se que, apesar da ideologia por trás do especifismo, compreendida esta como a sensação de superioridade em relação aos animais não humanos apenas por esses não pertencerem à mesma espécie que o homem, ser decorrente do pensamento secular greco-cristão, a maneira como se objetifica os animais só passaram a ser seriamente indagada após a publicação da obra "Libertação Animal" de Peter Singer (1946) em 1975. Singer denuncia o abuso aos animais de interesse zootécnico e de experimentação científica a partir do ponto de vista da senciência, onde o fato do animal ser capaz de sentir dor justifica seu direito a não sofrer (Sollund, 2017). Baseia-se, então, na não obrigatoriedade nutricional humana de consumir produtos de origem animal, mesmo que, na mesma obra, ele afirme que "julgamos os leões e os lobos como selvagens porque eles matam; mas eles têm de matar: se não o fizerem, morrem à fome", já que, nesse caso, haveriam interesses concorrentes em não padecer, reconhecendo, então, a necessidade desses animais de alimentarem-se de outros (inger, 2010).

A partir da proposta defendida em "Libertação Animal", estabeleceu-se a análise ética dos direitos dos animais que, para Francione (1995) ocorre a partir do direito mais básico implicado aos seres humanos que é o "de não ser propriedade, de não ser escravizado". Esse fundamento é vital para dissolução do especifismo, inclusive o seletivo que é classificado por Francione como uma "esquizofrenia moral" que se tem para com a proteção da vida dos animais domésticos enquanto se ignora o consumo de carne (Francione, 1995; Silva \& Kuhnen, 2015).

É importante notar que, apesar das críticas filosóficas, cães e gatos ainda possuem o status de "coisa" sob a visão jurídica brasileira, ao invés de serem considerados sujeitos de direitos. Todavia, já ocorrem no cenário jurídico do Brasil discussões quanto à guarda responsável de animais de companhia e da responsabilidade dos tutores nas famílias multiespécies (Dias \& Belchior, 2019). Nesses casos, o judiciário tende a interpretar as disputas da mesma maneira como se interpreta a questão da guarda de 
filhos menores de idade, levando em consideração os interesses dos animais de companhia (Levai, 2001; Vieira, 2016). Dessa maneira, os tutores deixam o papel de proprietários e passam a ser tidos como responsáveis legais, por escolha pessoal, de um ser vivo com predileções próprias, mesmo que dependente das pessoas que o tutelam. Além disso, apesar de ainda interpretar como propriedade o animal, a Lei 1.095/2019 decreta pena de reclusão de dois a cinco anos devido a abuso ou maus tratos a cães e gatos (Brasil, 2020).

Dentre os diversos atos abusivos contra os animais, inclui-se a negligência acerca do fornecimento de uma nutrição adequada (Solot, 1997). É possível, então, adotar no Direito a noção de família como vista pelo pós-humanismo, onde essas são formadas a partir de atos conscientes de escolha pela formação de laços de apego, sendo os cães e os gatos tidos como membros reais nesses lares enquanto animais de companhia, não assumindo papéis antropomorfizados como o de substituição de filhos (Charles, 2016; Irvine \& Cilia, 2017). Tamanha é a preocupação, que muitos tutores optam pela instalação de chips de rastreamento em seus cães de modo a evitar desaparecimentos (Gillespie \& Lawson, 2017; Mancini et al., 2012).

Contrapondo a visão direitista de Francione, é possível empregar a filosofia de Carol Gilligan (1936) ao analisar a relação de tutores para com seus cães e gatos ( $\underline{\mathrm{Li}}, 2002$; Tinoco \& Correia, 2010). Sob o ponto de vista de Gilligan, tem-se a ética do cuidado que valoriza as relações de afeto e resolução de conflitos de maneira pacífica a partir da linguagem (Dias, 2008). Logo, todo ser humano deveria ser cuidador do todo da natureza e de todos os animais pertencentes a esta (Gilligan, 1977; Grant, 2011; Zoboli, 2004). Portanto, devido ao processo de domesticação, tem-se uma dívida ética para com esses animais que tiveram seu comportamento, habitat e nutrição modificados pelo contato com o ser humano e que já não podem mais retornar a sua condição natural, dependendo quase exclusivamente de seus tutores para garantirem sua condição nutricional (Silva \& Kuhnen, 2015). E, como muitos tutores tendem a decidir sobre a alimentação de seus pets espelhando-se nas suas opções particulares de estilos de vida, eleva-se o número de pessoas que buscam, equivocadamente, alimentos veganos e vegetarianos para cães e gatos, além da obesidade dos tutores também ter sido relacionada ao excesso de acúmulo de gordura tecidual em seus respectivos animais de companhia (Brown, 2009; Knight \& Leitsberger, 2016).

Atualmente, os principais alimentos ofertados para animais de companhia são as rações secas e úmidas (latas ou sachês), produzidas de acordo com rigorosos parâmetros de excelência industrial e controle de qualidade (Zicker, 2008). As rações super premium suprem toda a necessidade nutricional da espécie para a qual foram desenvolvidas, havendo a segmentação das dietas segundo faixas etárias (neonatos, filhotes, adultos e idosos), raças (pequenas, médias, grandes e gigantes) e enfermidades específicas (como diabetes mellitus, insuficiência renal, urolitíase, alergia, gastroenteropatia, dermatopatia, cardiomiopatia e obesidade) (Bobeck, 2020; Freeman et al., 2011; Swanson et al., 2003).

Embora almejada por alguns tutores, a dieta "raw food", baseada no fornecimento de alimentos proteicos crus de origem animal e com a promessa de ser uma alimentação similar à encontrada pelos antepassados de seus animais na natureza, deve ser encarada com muita cautela nos dias de hoje (Fredriksson-Ahomaa et al., 2017; Tobie et al., 2015). Uma alimentação caseira jamais pode dispensar os cálculos nutricionais a serem realizados por nutricionista animal, que deve realizar a análise e o planejamento dietético de seu paciente canino ou felino. Outro erro comum é os tutores fornecerem restos alimentares de suas mesas, ou simplesmente as sobras de seus pratos, sendo que obstruções intestinais por corpos estranhos como ossos de frango podem ocasionar perfurações do trato digestório e inclusive levar o pet a óbito. Risco alto também ocorre quando não se atentam às condições sanitárias que envolvem a manipulação e o preparo de alimentos, ocasionando diversas toxinfecções alimentares como, por exemplo, a salmonelose (Buff et al., 2014; Davies et al., 2019; Kawauchi et al., 2014). Logo, as opções caseiras podem ser escolhas bastante perigosas, podendo representar uma dieta inadequada e desbalanceada. Portanto, na impossibilidade desta dieta ser desenvolvida e acompanhada por um profissional da área, o melhor é fornecer aos animais de companhia uma boa ração (

\section{Considerações finais}

Os filósofos estudados na presente pesquisa, Singer, Francione e Gilligan, propõem novos paradigmas para a relação cada vez mais humanizada entre tutores e seus animais de companhia. Isso, certamente, reflete em importantes mudanças nos hábitos alimentares dos pets, embora esse assunto 
divida opiniões. Entre as controvérsias a serem elucidadas nos futuros trabalhos, não seria impossível, pela óptica nutricional, que dietas veganas venham a ser desenvolvidas de maneira segura, como qualquer outra ração comercial. Por fim, é de suma importância que os tutores se responsabilizem pelos alimentos ofertados a seus animais de companhia, sempre levando em conta os aspectos morfofuncionais de cada espécie. Ademais, o acompanhamento médico veterinário com a realização de exames periódicos é fundamental para a checagem regular da saúde animal.

\section{Agradecimentos}

À FAPESP e ao CNPq pelo suporte financeiro concedido às pesquisadoras bolsistas.

À Profa. Dra. Flávia Maria de Oliveira Borges Saad, do Departamento de Zootecnia da Universidade Federal de Lavras, pela contribuição à revisão crítica do presente artigo.

À Profa. Dra. Maria Angélica Miglino, do Departamento de Cirurgia da Faculdade de Medicina Veterinária e Zootecnia da Universidade de São Paulo, pelo incentivo à pesquisa científica e apoio em diversos momentos.

\section{Referências bibliográficas}

Albright, K. M. (2002). The extension of legal rights to animals under a caring ethic: An ecofeminist exploration of Steven Wise's Rattling the Cage. Natural Resources Journal, 42(4), 915-938.

Bobeck, E. A. (2020). Nutrition and health: Companion animal applications: Functional nutrition in Livestock and Companion Animals to Modulate the Immune Response. Journal of Animal Science, 98(3), skaa035. https://doi.org/10.1093/jas/skaa035.

Bontempo, V. (2005). Nutrition and health of dogs and cats: evolution of petfood. Veterinary Research Communications, 29(2), 45-50. https://doi.org/10.1007/s11259-005-0010-8.

Brasil. (2020). Sancionada lei que aumenta punição para maus-tratos de animais. Governo do Brasil. Disponível em: <https://www.gov.br/pt-br/noticias/meio-ambiente-e-clima/2020/09/sancionada-leique-aumenta-punicao-para-maus-tratos-de-animais>. Acessado em 30/set. 2020.

Brown, W. Y. (2009). Nutritional and ethical issues regarding vegetarianism in the domestic dog. Recent Advances in Animal Nutrition in Australia, 17, 137-143.

Buff, P. R., Carter, R. A., Bauer, J. E., \& Kersey, J. H. (2014). Natural pet food: A review of natural diets and their impact on canine and feline physiology. Journal of Animal Science, 92(9), 3781-3791. https://doi.org/10.2527/jas.2014-7789.

Carciofi, A. C., \& Jeremias, J. T. (2010). Progresso científico sobre nutrição de animais de companhia na primeira década do século XXI. Revista Brasileira de Zootecnia, 39, 35-41.

Charles, N. (2016). Post-human families? Dog-human relations in the domestic sphere. Sociological Research Online, 21(3), 83-94. https://doi.org/10.5153/sro.3975.

Davies, R. H., Lawes, J. R., \& Wales, A. D. (2019). Raw diets for dogs and cats: a review, with particular reference to microbiological hazards. Journal of Small Animal Practice, 60(6), 329-339. https://doi.org/10.1111/jsap.13000.

Dias, M. R. M. S., \& Belchior, G. (2019). A guarda responsável dos animais de estimação na família multiespécie. Revista Brasileira de Direito Animal, 14(2). https://doi.org/10.9771/rbda.v14i2.33325.

Dias, T. L. P. (2008). A defesa dos rireitos dos animais sob uma ótica ecofeminista. Revista Brasileira de Direito Animal, 3(4). https://doi.org/10.9771/rbda.v3i4.10469.

Dodd, S. A. S., Cave, N. J., Adolphe, J. L., Shoveller, A. K., \& Verbrugghe, A. (2019). Plant-based (vegan) diets for pets: A survey of pet owner attitudes and feeding practices. PloS One, 14(1), e0210806. https://doi.org/10.1371/journal.pone.0210806.

Francione, G. L. (1995). Animal rights and animal welfare. Low, Culture and the Humanities, 6(1), 2436. https://doi.org/10.1177/1743872109348989.

Fredriksson-Ahomaa, M., Heikkilä, T., Pernu, N., Kovanen, S., Hielm-Björkman, A., \& Kivistö, R. (2017). Raw meat-based diets in dogs and cats. Veterinary Sciences, 4(3), 33. https://doi.org/10.3390/vetsci4030033. 
Freeman, L., Becvarova, I., Care, N., MacKay, C., Nguyen, P., Rama, B., \& Yathirai, S. (2011). WSAVA nutritional assessment guidelines. Journal of Feline Medicine \& Surgery, 13(7), 516-525. https://doi.org/10.1016/j.jfms.2011.05.009.

Gillespie, K., \& Lawson, V. (2017). 'My dog is my home': multispecies care and poverty politics in Los Angeles, California and Austin, Texas. Gender, Place \& Culture, 24(6), 774-793. https://doi.org/10.1080/0966369X.2017.1339021.

Gilligan, C. (1977). In a different voice: Women's conceptions of self and of morality. Harvard Educational Review, 47(4), 481-517. https://doi.org/10.17763/haer.47.4.g6167429416hg510.

Grant, C. (2011). Abolicionismo e direito animal-desconstruindo paradigmas: uma abordagem sob o prisma dos movimentos em prol dos direitos animais e da ética do cuidado. Revista Brasileira de Direito Animal, 6(8). https://doi.org/10.9771/rbda.v6i8.11063.

Irvine, L., \& Cilia, L. (2017). More-than-human families: Pets, people, and practices in multispecies households. Sociology Compass, 11(2), e12455. https://doi.org/10.1111/soc4.12455.

Kawauchi, I. M., Sakomura, N. K., Pontieri, C. F. F., Rebelato, A., Putarov, T. C., Malheiros, E. B., de OS Gomes, M., Castrillo, C., \& Carciofi, A. C. (2014). Prediction of crude protein digestibility of animal by-product meals for dogs by the protein solubility in pepsin method. Journal of Nutritional Science, 3. https://doi.org/10.1017/jns.2014.32.

Knight, A., \& Leitsberger, M. (2016). Vegetarian versus meat-based diets for companion animals. Animals, 6(9), 57. https://doi.org/10.3390/ani6090057.

Kuhnen, T. A. (2014). A ética do cuidado como teoria feminista. Simpósio Gênero e Políticas Públicas, 3.

Levai, L. F. (2001). Os animais sob a visão da ética. Congresso Ambiental do Ministério Público.

Li, H.-L. (2002). Animal research, non-vegetarianism, and the moral status of animals-Understanding the impasse of the animal rights problem. The Journal of Medicine and Philosophy, 27(5), 589-615. https://doi.org/10.1076/jmep.27.5.589.10322.

Mancini, C., Van Der Linden, J., Bryan, J., \& Stuart, A. (2012). Exploring interspecies sensemaking: dog tracking semiotics and multispecies ethnography. Proceedings of the 2012 ACM Conference on Ubiquitous Computing, 143-152.

Ramos, T. A., Kaelle, G. C. B., Risolia, L. W., do Rosário, B. C., de Oliveira, S. G., \& Félix, A. P. (2020). Impacto do enriquecimento ambiental sobre o comportamento de cães e digestibilidade da dieta em canil experimental. Archives of Veterinary Science, 25(3), 38-42. https://doi.org/10.5380/avs.v21i3.43902.

Rothgerber, H. (2014). Carnivorous cats, vegetarian dogs, and the resolution of the vegetarian's dilemma. Anthrozoös, 27(4), 485-498. https://doi.org/10.2752/089279314X14072268687844.

Saad, F. M. O. B., \& França, J. (2010). Alimentação natural para cães e gatos. Revista Brasileira de Zootecnia, 39(1), 52-59.

Silva, M. A., \& Kuhnen, T. A. (2015). Direitos e cuidado para a proteção da autonomia prática de animais não humanos. Revista Internacional Interdisciplinar, 12(1), 42-64. https://doi.org/10.5007/1807-1384.2015v12n1p42.

Singer, P. (2010). Libertação Animal. WMF Martins Fontes.

Sollund, R. (2017). Causes for speciesism: Difference, distance and denial. In Transnational Environmental Crime (pp. 75-96). Routledge.

Solot, D. (1997). Untangling the animal abuse web. Society \& Animals, 5(3), 257-265. https://doi.org/10.1163/156853097X00169.

Swanson, K. S., Schook, L. B., \& Fahey Jr, G. C. (2003). Nutritional genomics: implications for companion animals. The Journal of Nutrition, 133(10), 3033-3040. https://doi.org/10.1093/jn/133.10.3033.

Thatcher, C., Hand, M. S., \& Remillard, R. (2010). Small animal clinical nutrition: an iterative process. Small Animal Clinical Nutrition, 3-21.

Tinoco, I. A. P., \& Correia, M. L. A. (2010). Análise crítica sobre a declaração universal dos direitos dos animais. Revista Brasileira de Direito Animal, 5(7). https://doi.org/10.9771/rbda.v5i7.11043. 
Tobie, C., Péron, F., \& Larose, C. (2015). Assessing food preferences in dogs and cats: a review of the current methods. Animals, 5(1), 126-137. https://doi.org/10.3390/ani5010126.

Vieira, T. R. (2016). Biodireito, animal de estimação e equilíbrio familiar: apontamentos iniciais. Revista de Biodireito e Direito dos Animais, 2(1), 179-195. https://doi.org/10.26668/IndexLawJournals/2525-9695/2016.v2i1.280.

Zafalon, R. V. A., Risolia, L. W., Vendramini, T. H. A., Ayres Rodrigues, R. B., Pedrinelli, V., Teixeira, F. A., Rentas, M. F., Perini, M. P., Alvarenga, I. C., \& Brunetto, M. A. (2020). Nutritional inadequacies in commercial vegan foods for dogs and cats. PloS One, 15(1), e0227046. https://doi.org/10.1371/journal.pone.0227046.

Zaghini, G., \& Biagi, G. (2005). Nutritional peculiarities and diet palatability in the cat. Veterinary Research Communications, 29(2), 39-44. https://doi.org/10.1007/s11259-005-0009-1.

Zicker, S. C. (2008). Evaluating pet foods: how confident are you when you recommend a commercial pet food? Topics in Companion Animal Medicine, 23(3), 121-126. https://doi.org/10.1053/j.tcam.2008.04.003.

Zoboli, E. L. C. P. (2004). A redescoberta da ética do cuidado: o foco e a ênfase nas relações. Revista da Escola de Enfermagem da USP, 38(1), 21-27. https://doi.org/10.1590/S008062342004000100003.

Zoran, D. L. (2002). The carnivore connection to nutrition in cats. Journal of the American Veterinary Medical Association, 221(11), 1559-1567. https://doi.org/10.2460/javma.2002.221.1559.

Histórico do artigo:

Recebido: 13 de agosto de 2021

Aprovado: 13 de setembro de 2021

Disponível on-line: 12 de novembro de 2021
Licenciamento: Este artigo é publicado na modalidade Acesso Aberto sob a licença Creative Commons Atribuição 4.0 (CC-BY 4.0), a qual permite uso irrestrito, distribuição, reprodução em qualquer meio, desde que o autor e a fonte sejam devidamente creditados. 